\title{
Underlying mechanism of Sirt1 on apoptosis and extracellular matrix degradation of osteoarthritis chondrocytes
}

\author{
DONG-SHENG HE, XIAO-JIAN HU, YI-QI YAN and HUI LIU \\ Department of Orthopedics, Xinyu City People's Hospital, Xinyu, Jiangxi 338025, P.R. China
}

Received January 15, 2016; Accepted February 6, 2017

DOI: $10.3892 / \mathrm{mmr} .2017 .6659$

\begin{abstract}
The study investigated the effects and underlying mechanisms of silent information regulation of transcription 1 (Sirt1) action on apoptosis in chondrocytes and degradation of the extracellular matrix. Cartilage tissue samples were derived from knee arthroplasty of patients with osteoarthritis (OA). The three groups were as follows: Control, resveratrol (Res) and Res+small interfering (si)RNA (Res+siRNA Sirt1). The level of Sirt1 protein expression significantly increased in the Res group $(1.03 \pm 0.10)$ compared with the control $(0.22 \pm 0.03)$ and Res+siRNA $(0.18 \pm 0.01)$ groups (both $\mathrm{P}<0.05)$. Early and late stage cell apoptosis rates decreased in the Res group and increased in the Res+siRNA group (both $\mathrm{P}<0.05$ ). B-cell lymphoma 2 (Bcl-2) expression levels were upregulated and Bcl-2-associated X protein (Bax) expression levels were downregulated in the Res group compared with the control group. Protein expression levels of MMP1 and MMP13 and the phosphorylation levels of extracellular signal regulated kinase (ERK), c-Jun N-terminal kinase (JNK) and p38 were downregulated in the Res group and upregulated in the Res+siRNA group. In conclusion, upregulation of Sirt1 expression may inhibit OA chondrocyte apoptosis and extracellular matrix degradation by increasing Bcl-2 expression and decreasing Bax, MMP1 and MMP13 expression, via downregulation of p38, JNK and ERK phosphorylation.
\end{abstract}

\section{Introduction}

Osteoarthritis (OA) is a chronic and degenerative joint disease that occurs frequently in elderly individuals (1). The disability rate of $\mathrm{OA}$ among the older population ranks only second to cardiovascular diseases (2). Articular cartilage damage and osteophyte are the primary pathological features of OA, which

Correspondence to: Professor Dong-Sheng He, Department of Orthopedics, Xinyu City People's Hospital, 3 Xinxing Road, Xinyu, Jiangxi 338025, P.R. China

E-mail: hdshospital@foxmail.com

Key words: nicotinamide-adenine dinucleotide-dependent protein deacetylase sirtuin-1, osteoarthritis, apoptosis, degradation, signaling pathway is associated with gender, obesity, trauma, inflammation and genetic factors; however, ageing is a primary factor (3). External and biological factors lead to the imbalance of chondrocytes, extracellular matrix and subchondral bone (4-6). Present studies have primarily focused on how cartilago articularis maintains a dynamic equilibrium of cell proliferation and extracellular matrix metabolism.

Silent information regulation of transcription 1 (Sirt1) is the most extensively studied protein of the sirtuin family. Sirt1 is a type of conservative protein and an $\mathrm{NAD}^{+}$-dependent histone deacetylase that exists in all mammalian somatic cells. Sirt1 is involved in diseases, including neural degenerative disease, diabetes, tumor, inflammation and senility (7). A previous study demonstrated that Sirt1 is expressed in human articular cartilage tissues and cells. However, Sirt1 expression levels are reduced in the chondrocytes of patients with OA. Upregulating Sirt1 expression levels promotes the expression levels of cartilage specific genes and survival of chondrocytes, and inhibits apoptosis of chondrocytes significantly (8). Another study revealed that upregulating Sirtl activity an OA mouse model reduces the expression levels of inflammatory mediators including matrix metalloproteinase (MMP) 13 and inducible nitric oxide synthase (iNOS) in chondrocytes, thus inhibits cartilage degeneration in mice (9). The expression levels of Sirt1 have significant inhibitory effects on the occurrence and development of OA; however, the underlying mechanisms of action remain unclear. Based on the above observations, the present study investigated the effects and underlying mechanisms of action of Sirt1 on apoptosis of chondrocytes and degradation of the extracellular matrix in patients with OA.

\section{Materials and methods}

Patients. Cartilage tissues were obtained from knee arthroplasty of 28 patients with OA (age, 56-86 years; mean age, 69 years; males, 12; females, 16) from November 2014 to November 2015. According to the OA diagnostic criteria developed by the American Institute of Rheumatoid Arthritis in 2008, the OA patients were diagnosed by clinical examination and X-ray plain films. Informed consent was obtained from the patients, and the trial was approved by the ethics committee of Xinyu City People's Hospital (Xinyu, China).

Reagents and kits. The following primary antibodies were used: Rabbit polyclonal anti-Sirt1 (cat. no. bs-2257R; 
Beijing Boosen Biological Technology Co., Ltd., Beijing, China); rabbit monoclonal anti-apoptosis regulator B-cell lymphoma 2 (Bcl-2; cat. no. 1017-1), anti-apoptosis regulator Bcl-2-associated X protein (Bax; cat. no. 1063-1), anti-extracellular signal regulated kinase 1/2 (ERK1/2; cat. no. 8663-1), anti-phosphorylated (p)-ERK1/2 (cat. no. 1418-1), anti-c-Jun N-terminal kinase (JNK; cat. no. 3496-1), anti-p-JNK (cat. no. 2155-1), anti-p-p38 mitogen activated protein kinase (MAPK; cat. no. 5359-1), anti-p38 (cat. no. 2132-1; all purchased from Epitomics, Burlingame, CA, USA); rabbit polyclonal anti-MMP1 (cat. no. S1023) and MMP13 (cat. no. 1923-1; Abcam, Cambridge, UK). Mouse anti-GAPDH (cat. no. AG019) and an Annexin V-propidium iodide (PI; cat. no. C1063) double staining flow cytometry detection kit were purchased from Beyotime Institute of Biotechnology (Haimen, China), resveratrol (Res) was obtained from Sigma-Aldrich; Merck Millipore (Darmstadt, Germany), Sirtl small interfering (si)RNA was purchased from Shanghai Pharmaceutical Group Co., Ltd. (Shanghai, China), and type II collagenase, fetal bovine serum (FBS) and Dulbecco's modified Eagle's medium/nutrient mixture F-12 (DMEM/F-12) were obtained from Gibco; Thermo Fisher Scientific, Inc. (Waltham, MA, USA). The XRS ChemiDoc ${ }^{\mathrm{TM}}$ gel imaging system was purchased from Bio-Rad Laboratories, Inc. (Hercules, CA, USA) and the FACSCalibur ${ }^{\mathrm{TM}}$ flow cytometry (BD Biosciences, Franklin Lakes, NJ, USA).

Preparation and grouping of chondrocytes. Under sterile conditions, the OA cartilage tissue was washed and cut into $1-\mathrm{mm}^{3}$ sections using ophthalmology scissors. The tissues were digested with $0.25 \%$ trypsin for $30 \mathrm{~min}$, following which cells were digested $0.2 \%$ collagenase for $2 \mathrm{~h}$. Once the single cell suspension was obtained, cells were cultured in DMEM/F-12 supplemented with $10 \% \mathrm{FBS}$ at $37^{\circ} \mathrm{C}$ and $5 \% \mathrm{CO}_{2}$. After 4-5 days, cells began to fuse and 2-3 generation cells were used.

Grouping. Chondrocytes at $80 \%$ fusion degree were randomly divided into 3 groups: Control (cultured with DMEM/F-12, without any external stimulus); Res (10 $\mu \mathrm{M}$ resveratrol treatment) and Res+siRNA [10 $\mu \mathrm{M}$ resveratrol+siRNA Sirt1, transfected using Lipofectamine ${ }^{\circledR} 2000$ (Thermo Fisher Scientific, Inc.)]. All groups were cultured for $4 \mathrm{~h}$.

Expression levels of Sirtl in OA chondrocytes by reverse transcription-semiquantitative polymerase chain reaction (RT-sqPCR) analysis. Total RNA was extracted using TRIzol ${ }^{\circledR}$ reagent (Invitrogen; Thermo Fisher Scientific, Inc.) according to the manufacturer's protocol. The primers used were as follows: Sirt1 primer: Forward, 5'-TGGACTCCACGACGT ACT-3' and reverse, 5'-TCTCCTGGGAGGCATAGACC-3' (122 bp) for Sirt1; and forward, 5'-AGCCACATCGCTCAG ACA-3' and reverse, 5'-TCTCCTGGGAGGCATAGACC-3' (314 bp) for GAPDH. RNA was transcribed into cDNA and amplified by PCR to obtain $5 \mu 1$ amplification products using a one-step qPCR kit (cat. no. DRR064A; Takara Bio, Inc., Otsu, Japan). PCR was performed at $95^{\circ} \mathrm{C}$ for $5 \mathrm{~min}$ followed by 40 cycles at $95^{\circ} \mathrm{C}$ for $30 \mathrm{sec}$, at $55^{\circ} \mathrm{C}$ for $30 \mathrm{sec}$, at $72^{\circ} \mathrm{C}$ for $45 \mathrm{sec}$, and at $72^{\circ} \mathrm{C}$ for $10 \mathrm{~min}$ for a final extension. The PCR-amplified products were verified using a $1.2 \%$ agarose gel at $100 \mathrm{~V}$ for $20 \mathrm{~min}$ and the results were analyzed by gel imaging and analysis system (WE-9413B; Beijing Liuyi Instrument Company, Beijing, China).

Cell viability detection by MTT assay. A total of $20 \mu 15 \mathrm{mg} / \mathrm{ml}$ MTT (cat. no. KA1606; Abnova, Taipei, Taiwan) and $150 \mu \mathrm{l}$ dimethyl sulfoxide was added to cells for $4 \mathrm{~h}$, following which the optical density (OD) value was detected at a wavelength of $560 \mathrm{~nm}$ using a microplate reader. Cell viability $(\%)=(\mathrm{OD}$ value of experimental group/OD value of control group)x100.

Cell apoptosis analysis by Annexin V-propidium iodide (PI) double staining flow cytometry. Cells were digested with $0.25 \%$ trypsin (no EDTA) and collected, following which $500 \mu \mathrm{l}$ Binding Buffer was added. A total of $5 \mu \mathrm{l}$ Annexin V-fluorescein isothyanate (FITC) was added to the cells, following which $5 \mu \mathrm{l}$ PI was added. Avoidance response was performed at room temperature for 5-15 min and it was subsequently detected by flow cytometry after $1 \mathrm{~h}$ and analyzed by the software of CellQuest (BD Biosciences).

Western blotting. Proteins were extracted by centrifugation with $13,400 \mathrm{x} g$ at 4 for $5 \mathrm{~min}$, following which lysis buffer (cat. no. P0013; Beyotime Institute of Biotechnology) was added to obtain the total protein. Protein concentration was measured using a Bicinchoninic Acid assay kit. Equal quantity of protein per lane $(50 \mu \mathrm{g})$ was separated by $4 \%$ SDS-PAGE gel and subsequently transferred onto PVDF membranes. Membranes were incubated with primary antibodies overnight at $4^{\circ} \mathrm{C}$. Following washing with PBS, membranes were incubated with secondary antibodies at room temperature for 1 to $2 \mathrm{~h}$. Following this, the membrane was removed and washed and an Enhanced Chemiluminescence reagent (cat. no. WBKLS0500; Merck Millipore) was added. Densitometry was performed using Quantity One version 4.62 software (Bio-Rad Laboratories, Inc.).

Statistical analysis. Data are expressed as the mean \pm standard deviation. Every experiment was repeated three times. Independent Student's t-test was used to determine differences between groups. $\mathrm{P}<0.05$ was considered to indicate a statistically significant difference. All analyses were performed using SPSS software version 17.0 (SPSS, Inc., Chicago, IL, USA).

\section{Results}

Sirtl protein and mRNA expression levels. As presented in Fig. 1, Sirtl protein expression levels were significantly increased in the Res group $(1.03 \pm 0.10)$ compared with the control $(0.22 \pm 0.03)$ and Res+siRNA groups $(0.18 \pm 0.01$; both $\mathrm{P}<0.05)$. Sirt1 mRNA expression levels were significantly increased in the Res group $(0.98 \pm 0.08)$ compared with the control $(0.30 \pm 0.03)$ and Res+siRNA groups $(0.08 \pm 0.01$; both $\mathrm{P}<0.05)$.

Bax and Bcl-2 protein expression levels. The protein expression levels of Bax were downregulated, and Bcl-2 upregulated, in the Res group compared with the control group. However, Bax and Bcl-2 protein expression levels increased in the Res+siRNA group compared with the Res group (Table I; Fig. 2).

MMP1 and MMP13 protein expression levels. Compared with the control group, the protein expression levels of MMP1 and 
Table I. Cell viability, apoptosis and protein expression levels in osteoarthritis chondrocytes.

\begin{tabular}{|c|c|c|c|}
\hline Parameters & Control & Res & Res+siRNA \\
\hline Cell viability $\%^{a-c}$ & $89.45 \pm 8.72$ & $94.38 \pm 5.06$ & $70.76 \pm 7.38$ \\
\hline \multicolumn{4}{|l|}{ Cell apoptosis \% } \\
\hline Early $^{\mathrm{a}-\mathrm{c}}$ & $2.83 \pm 0.22$ & $1.70 \pm 0.14$ & $5.88 \pm 0.59$ \\
\hline Late $^{\mathrm{a}-\mathrm{c}}$ & $4.30 \pm 0.41$ & $3.10 \pm 0.34$ & $17.57 \pm 1.69$ \\
\hline $\mathrm{Bax} / \mathrm{GAPDH}^{\mathrm{a}, \mathrm{c}}$ & $0.45 \pm 0.04$ & $0.23 \pm 0.02$ & $0.55 \pm 0.04$ \\
\hline Bcl-2/GAPDH ${ }^{\mathrm{a}, \mathrm{c}}$ & $0.34 \pm 0.02$ & $1.02 \pm 0.10$ & $0.22 \pm 0.02$ \\
\hline MMP1/GAPDH ${ }^{\mathrm{a}, \mathrm{c}}$ & $0.48 \pm 0.04$ & $0.30 \pm 0.02$ & $0.89 \pm 0.08$ \\
\hline MMP13/GAPDH ${ }^{\mathrm{a}, \mathrm{c}}$ & $0.44 \pm 0.04$ & $0.29 \pm 0.02$ & $0.94 \pm 0.04$ \\
\hline p-ERK/ERK ${ }^{\mathrm{a}-\mathrm{c}}$ & $0.51 \pm 0.04$ & $0.14 \pm 0.01$ & $0.56 \pm 0.04$ \\
\hline p-JNK/JNK ${ }^{\mathrm{a}-\mathrm{c}}$ & $1.03 \pm 0.10$ & $0.29 \pm 0.02$ & $1.28 \pm 0.13$ \\
\hline p-p38/p38 $8^{\mathrm{a}-\mathrm{c}}$ & $0.99 \pm 0.08$ & $0.28 \pm 0.02$ & $1.32 \pm 0.11$ \\
\hline
\end{tabular}

${ }^{a} \mathrm{P}<0.05$ control vs. Res; ${ }^{\mathrm{b}} \mathrm{P}<0.05$ control vs. Res+siRNA; ${ }^{\mathrm{c}} \mathrm{P}<0.05$; Res vs. Res+siRNA. Bcl-2, B-cell lymphoma 2; BAx, Bcl-2-associated X protein; MMP, matrix metalloproteinase; ERK, extracellular signal regulated kinase; JNK, c-Jun N-terminal kinase; p, phosphorylated; Res, resveratrol; siRNA, small interfering RNA.

A

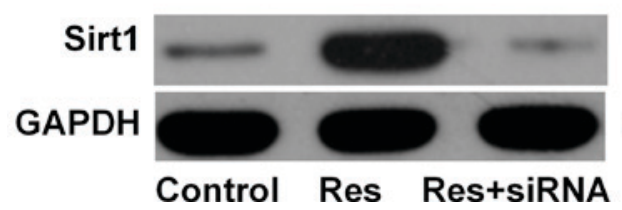

B

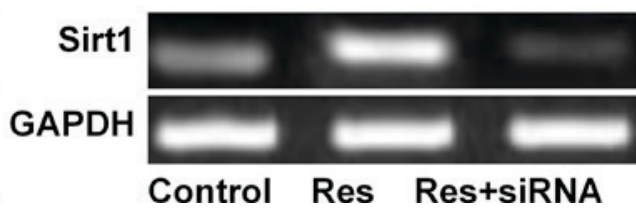

Figure 1. Protein and mRNA expression levels. (A) Protein and (B) mRNA expression levels of Sirt1. Sirt1, silent information regulation of transcription 1.

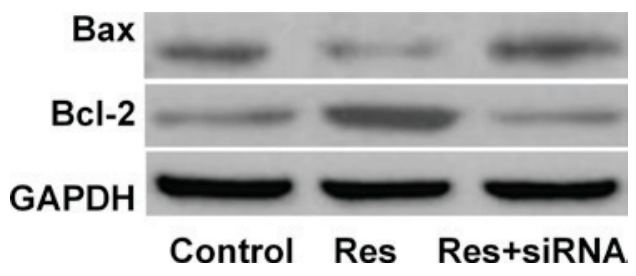

Figure 2. Protein expression levels of Bax and Bcl-2. Bcl-2, B-cell lymphoma 2; Bax, B-cell lymphoma 2-associated X protein.

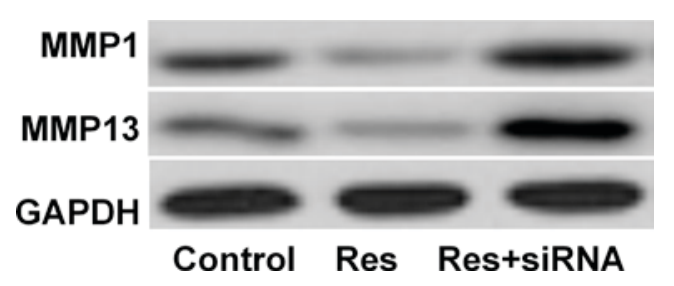

Figure 3. Protein expression levels of MMPs. MMP, matrix metalloproteinase

MMP13 were downregulated in the Res group and upregulated in the Res+siRNA group, and were significantly different between the Res and Res+siRNA groups (Table I; Fig. 3).

MAPK signal protein expression. Compared with the control group, the phosphorylation levels of ERK, JNK and p38 were

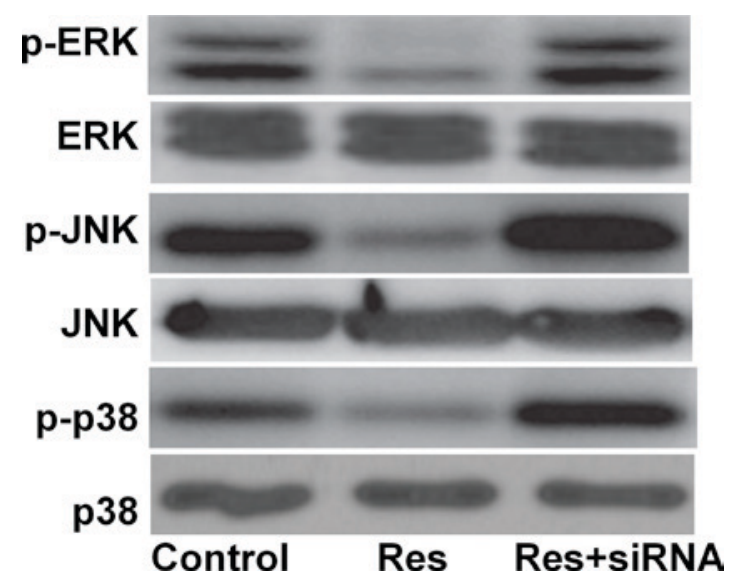

Figure 4. Protein expression levels of mitogen activated protein kinase signaling pathway proteins. ERK, extracellular signal regulated kinase; $\mathrm{p}$, phosphorylated; JNK, c-Jun N-terminal kinase; Res, resveratrol; siRNA, small interfering RNA.

decreased in the Res group and increased in the Res+siRNA group. They were additionally significantly different between the Res and Res+siRNA groups (Table I; Fig. 4).

Detection of cell viability and apoptosis. Compared with the control group (89.45\%; Fig. 5A), cell viability was significantly increased in the Res group (94.38\%; Fig. 5B), and significantly 

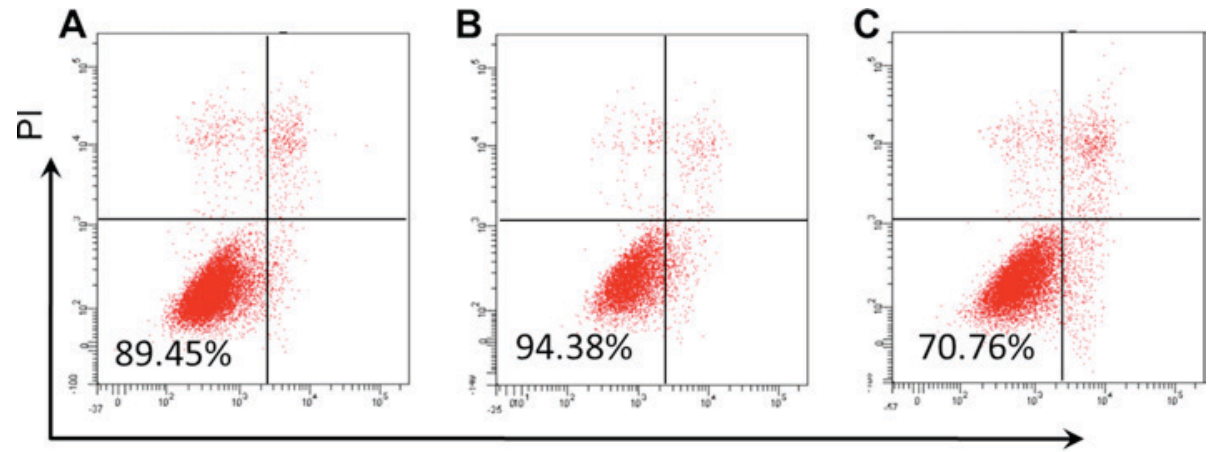

Annexin $\mathrm{V}$

Figure 5. Apoptosis of osteoarthritis cartilage cells as assessed by Annexin V/PI staining. (A) Control, (B) Res and (C) Res+small interfering (si)RNA groups. Res, resveratrol; PI, propidium iodide.

reduced in the Res+siRNA group (70.76\%; Fig. 5C). Cell apoptosis rates decreased in the Res group, whereas they increased in the Res+siRNA group (both $\mathrm{P}<0.05$; Table I; Fig. 5).

\section{Discussion}

The sirtuin 2 (Sir2) gene family, which exists in the chromatin of yeast, is widely associated with numerous physiological and pathological processes. Sirt1, a homologue of Sir2, is associated with apoptosis, the cell cycle, cell energy metabolism, lipid accumulation and cell aging (10). Sirt1 has been demonstrated to serve important roles in extracellular matrix synthesis and cell survival, and has anti-inflammatory actions in human OA chondrocytes $(11,12)$. Fujita et al (8) demonstrated that expression levels of Sirt1 are decreased in OA cartilage cells compared with healthy articular cartilage cells. Expression levels of transcription factor Sox9 was significantly promoted in OA chondrocytes transfected with wild-type Sirt1, and in chondrocytes transfected with mutant Sirt1, its expression levels reduced. Additionally, Gagarina et al (13) reported that Sirt1 may promote OA cartilage-specific gene expression and slow OA progression. Furthermore, Gabay et al (14) demonstrated that Sirt1 knockout altered cartilage expression, increased apoptosis and acceleration cartilage degeneration in mice. Sirt1 is able to block chondrocyte apoptosis mediated by tumor necrosis factor- $\alpha$ (15). Therefore, chondrocyte apoptosis may be significantly suppressed by increasing expression levels of Sirt1, which reduces the degree of cartilage degeneration. The present study used resveratrol treatment and siRNA interference to investigate viability and apoptosis of OA cartilage cells. The results demonstrated that cartilage cell viability was promoted and apoptosis was reduced significantly on OA chondrocytes in the Res group compared with the control group. Additionally, cartilage cell viability was markedly reduced and apoptosis was significantly increased by siRNA Sirt1 transfection, compared with the control group. These results illustrated that increased Sirt1 expression levels serve an inhibitory effect on apoptosis in OA chondrocytes. Therefore, the present study further investigated the protein expression levels of Bax and $\mathrm{Bcl}-2$ in each group of chondrocytes. The results revealed that when Sirtl expression levels were increased, Bax expression levels decreased and Bcl-2 expression levels increased. With the intervention of siRNA Sirt1 and Res treatment, protein expression levels of Bax increased and Bcl-2 expression levels decreased, consistent with the above results from cell viability and apoptosis assays. Takayama et al (16) previously demonstrated that, by regulating Bax and Bcl-2 levels, Sirt1 resists nitric oxide-induced chondrocyte apoptosis. This further illustrated that during OA chondrocyte apoptosis, inhibition of Sirt1 is achieved by regulating Bax and $\mathrm{Bcl}-2$ expression levels.

Cartilage degeneration is a key pathological feature of OA, and is mediated by an imbalance of cartilage apoptosis and extracellular matrix degradation, exacerbating OA progression. MMPs, a $\mathrm{Zn}^{2+}$ dependent protease superfamily, are the most important proteolytic enzymes in the extracellular matrix degrading process. MMPs are present in $>25$ species, and the most critical ones in OA are collagenases, including MMP1 and MMP13. MMP1 and MMP13 may degrade cartilage-specific extracellular matrix components including collagen type II. MMP1 may degrade proteoglycans and collagens type I and III. The degradation of MMP13 was 10 times greater compared with MMP1, which may degrade the type II collagen triple helix structure, contributing to the hydrolysis of other proteases. It has previously been reported that in the process of OA development, the content and activity of MMP1 and MMP13 were increased and enhanced. When their activity or expression levels were suppressed, collagen synthesis was promoted, and cartilage degeneration was inhibited (17,18). Meanwhile, Li et al (9) demonstrated that resveratrol may inhibit cartilage degeneration in OA mice by increasing Sirt1 activity and decreasing MMP13 and iNOS expression levels. Matsuzaki et al (19) demonstrated that Sirt1-conditional knockout mice were more likely to develop OA compared with 8-week old wild-type C57BL6/J mice, and exhibited increased expression levels of collagen $X$ and MMP13. Therefore, based on this, the present study examined MMP1 and MMP13 expression levels in each group of cells by western blot analysis. The results revealed that compared with the control group, upregulation of Sirt1 expression levels may significantly inhibit MMP1 and MMP13 expression levels in OA chondrocytes, and with the intervention of siRNA on Sirt1 expression levels, MMP1 and MMP13 expression levels were significantly downregulated. This indicated that MMP1 and MMP13 expression levels may be significantly inhibited by upregulating the expression levels of Sirt1 in OA chondrocytes, 
which may reduce extracellular matrix degradation and mitigate cartilage degeneration.

The process of OA development is subject to a variety of inflammatory cytokines and mechanical stress stimulation. Stimulation signals are transmitted to various transcription factors via signal transduction pathways, to regulate chondrocyte apoptosis and extracellular matrix degradation. MAPK is the most important signaling pathway in mediating cartilage degeneration damage (20). The MAPK signaling pathway regulates cell withered death, proliferation, hypertrophy, inflammation and other physiological response in a three-level manner: Inducing MAPKKK phosphorylation, activating MAPKK and finally phosphorylating MAPK, which enters the nucleus, mediated by a class of serine/threonine protein kinases present in eukaryotic cells. There are eight MAPK subfamilies involved in the pathogenesis of OA: JNK, p38 and ERK. Primarily p38 MAPK mediates inflammatory pathways in OA chondrocytes, inducing the expression of MMP13 and causing type II collagen degradation $(21,22)$. Additionally, it has been reported that the p38 inhibitor may significantly reduce cartilage degeneration in a rat model of $\mathrm{OA}$, and inhibit the expression levels of inflammatory factors (23). JNK is involved in the regulation of MMP3 and MMP13 expression levels by regulating its downstream target proteins activator protein 1, c-Fos and c-Jun, and is additionally involved in apoptosis of chondrocytes. Yang et al (24) demonstrated that the JNK inhibitor SP600125 significantly inhibits NO-induced upregulation of MMP13 chondrocytes (21). Yoon et al (25) reported that stimulation of transforming growth factor- $\alpha$ in chondrocytes leads to increased JNK activity; JNK is involved in apoptosis and reduces activity of the apoptosis proteins Bcl-2 and Bcl-2 family apoptosis regulator. The ERK signaling pathway is primarily associated with chondrocyte proliferation and hypertrophy; however, research has additionally reported that ERK inhibitors combined with hyaluronic acid significantly reduced ERK phosphorylation levels, therefore reducing the expression levels of MMP13 and delaying hypertrophic chondrocyte and cartilage degeneration (26). Therefore, by inactivating the MAPK signaling pathway, apoptosis and degradation of the extracellular matrix of cartilage cells may be significantly inhibited, relieving cartilage degeneration. Therefore, the present study examined the levels of p38, JNK and ERK phosphorylation following Sirt1 overexpression or reduced expression in OA chondrocytes by western blot. The results indicated Sirt1 overexpression led to reduced phosphorylation of p38, JNK and ERK in OA chondrocytes, whereas p38, JNK and ERK phosphorylation levels were increased when OA chondrocytes were treated with combined Sirtl siRNA and resveratrol. Bai et al (27) demonstrated that resveratrol may inhibit pulmonary vascular endothelial cell apoptosis by upregulating Sirt1 expression levels and reducing p38 MAPK activity in burn-induced mice, whereas Sirt1 siRNA promotes apoptosis caused by burning and increases p38 MAPK activity (27). Becatti et al (28) demonstrated that myocardial apoptosis injury caused by ischemia-reperfusion, oxidative stress injury and mitochondrial dysfunction may be inhibited by Sirtl overexpression, including reduction of p38 and JNK phosphorylation levels, thus increasing ERK phosphorylation. The results of the present study illustrated that increased Sirt1 expression levels in OA chondrocytes may significantly reduce p38, JNK and ERK phosphorylation levels, thus inhibiting chondrocyte apoptosis and extracellular matrix degradation. The level of ERK phosphorylation is inconsistent with the results of Becatti et al (28), potentially due to the fact that at different time points, Sirt1 promotes cell survival by upregulating ERK phosphorylation levels and additionally inhibits the secretion of MMPs into extracellular matrix degradation by decreasing the level of inhibition of ERK phosphorylation. Therefore, further examination of phosphorylation levels across time points is required.

In conclusion, upregulation of Sirt1 expression levels may inhibit OA chondrocyte apoptosis and extracellular matrix degradation by increasing of Bcl-2 expression levels and decreasing Bax, MMP1 and MMP13 expression levels. This may be achieved by downregulating phosphorylation levels of p38, JNK and ERK.

\section{References}

1. Musumeci G, Leonardi R, Carnazza ML, Cardile V, Pichler K, Weinberg AM and Loreto C: Aquaporin 1 (AQP1) expression in experimentally induced osteoarthritic knee menisci: An in vivo and in vitro study. Tissue Cell 45: 145-152, 2013.

2. Felson DT and Zhang Y: An update on the epidemiology of knee and hip osteoarthritis with a view to prevention. Arthritis Rheum 41: 1343-1355, 1988.

3. Lee AS, Ellman MB, Yan D, Kroin JS, Cole BJ, van Wijnen AJ and Im HJ: A current review of molecular mechanisms regarding osteoarthritis and pain. Gene 527: 440-447, 2013.

4. Musumeci G, Szychlinska MA and Mobasheri A: Age-related degeneration of articular cartilage in the pathogenesis of osteoarthritis: Molecular markers of senescent chondrocytes. Histol Histopathol 30: 1-12, 2015.

5. Zhang X, Xu X, Xu T and Qin S: $\beta$-Ecdysterone suppresses interleukin-1 $\beta$-induced apoptosis and inflammation in rat chondrocytes via inhibition of NF- $\kappa$ B signaling pathway. Drug Dev Res 75: 195-201, 2014.

6. Zhang XH, Xu XX and Xu T: Ginsenoside Ro suppresses interleukin-lbeta-induced apoptosis and inflammation in rat chondrocytes by inhibiting NF- $\mathrm{KB}$. Chin J Nat Med 13: 283-289, 2015.

7. Yu XY, Zhang YL, Cao YJ and Liu CF: Histone deacetylase SIRT1 and cell autophagy. Chinese J Pathophysiol 29: 1520-1524, 2013.

8. Fujita N, Matsushita T, Ishida K, Kubo S, Matsumoto T, Takayama K, Kurosaka M and Kuroda R: Potential involvement of SIRT1 in the pathogenesis of osteoarthritis through the modulation of chondrocyte gene expressions. J Orthop Res 29: 511-515, 2011.

9. Li W, Cai L, Zhang Y, Cui L and Shen G: Intra-articular resveratrol injection prevents osteoarthritis progression in a mouse model by activating SIRT1 and thereby silencing HIF- $2 \alpha$. J Orthop Res 33: 1061-1070, 2015.

10. Seo JS, Moon MH, Jeong JK, Seol JW, Lee YJ, Park BH and Park SY: SIRT1, a histone deacetylase, regulates prion protein-induced neuronal cell death. Neurobiol Aging 33: 1110-1120, 2012

11. Dvir-Ginzberg M and Steinmeyer J: Towards elucidating the role of SirT1 in osteoarthritis. Front Biosci (Landmark Ed) 18: 343-355, 2013.

12. Lim HD, Kim YS, Ko SH, Yoon IJ, Cho SG, Chun YH, Choi BJ and Kim EC: Cytoprotective and anti-inflammatory effects of melatonin in hydrogen peroxide-stimulated $\mathrm{CHON}-001$ human chondrocyte cell line and rabbit model of osteoarthritis via the SIRT1 pathway. J Pineal Res 53: 225-237, 2012.

13. Gagarina V, Gabay O, Dvir-Ginzberg M, Lee EJ, Brady JK, Quon MJ and Hall DJ: SirT1 enhances survival of human osteoarthritic chondrocytes by repressing protein tyrosine phosphatase $1 \mathrm{~B}$ and activating the insulin-like growth factor receptor pathway. Arthritis Rheum 62: 1383-1392, 2010.

14. Gabay O, Zaal KJ, Sanchez C, Dvir-Ginzberg M, Gagarina V, Song Y, He XH and McBurney MW: Sirt1-deficient mice exhibit an altered cartilage phenotype. Joint Bone Spine 80: 613-620, 2013. 
15. Oppenheimer H, Kumar A, Meir H, Schwartz I, Zini A, Haze A, Kandel L, Mattan Y, Liebergall M and Dvir-Ginzberg M: Set7/9 impacts COL2A1 expression through binding and repression of SirT1 histone deacetylation. J Bone Miner Res 29: 348-360, 2014.

16. Takayama K, Ishida K, Matsushita T, Fujita N, Hayashi S, Sasaki K, Tei K, Kubo S, Matsumoto T, Fujioka H, et al: SIRT1 regulation of apoptosis of human chondrocytes. Arthritis Rheum 60: 2731-2740, 2009.

17. Lim NH, Meinjohanns E, Meldal M, Bou-Gharios G and Nagase H: In vivo imaging of MMP-13 activity in the murine destabilised medial meniscus surgical model of osteoarthritis. Osteoarthritis Cartilage 22: 862-868, 2014.

18. Wu H, Du J and Zheng Q: Expression of MMP-1 in cartilage and synovium of experimentally induced rabbit ACLT traumatic osteoarthritis: Immunohistochemical study. Rheumatol Int 29 31-36, 2008.

19. Matsuzaki T, Matsushita T, Takayama K, Matsumoto T, Nishida K, Kuroda R and Kurosaka M: Disruption of Sirt1 in chondrocytes causes accelerated progression of osteoarthritis under mechanical stress and during ageing in mice. Ann Rheum Dis 73: 1397-1404, 2014.

20. Chowdhury TT, Salter DM, Bader DL and Lee DA: Signal transduction pathways involving p38 MAPK, JNK, NFkappaB and AP-1 influences the response of chondrocytes cultured in agarose constructs to IL-1beta and dynamic compression. Inflamm Res 57: 306-313, 2008.

21. Lim H and Kim HP: Matrix metalloproteinase-13 expression in IL-1 $\beta$-treated chondrocytes by activation of the p38 MAPK/c-Fos/AP-1 and JAK/STAT pathways. Arch Pharm Res 34: 109-117, 2011.
22. Wei L, Sun XJ, Wang Z and Chen Q: CD95-induced osteoarthritic chondrocyte apoptosis and necrosis: Dependency on p38 mitogen-activated protein kinase. Arthritis Res Ther 8: R37, 2006.

23. Brown KK, Heitmeyer SA, Hookfin EB, Hsieh L, Buchalova M, Taiwo YO and Janusz MJ: P38 MAP kinase inhibitors as potential therapeutics for the treatment of joint degeneration and pain associated with osteoarthritis. J Inflamm (Lond) 5: 22, 2008

24. Yang L, Guo A and Gu JC: c-Jun N-terminal kinase and nuclear factor $\kappa \mathrm{B}$ mediate nitric oxide-induced expression of matrix metalloproteinase-13. Int Orthop 35: 1261-1266, 2011.

25. Yoon HS and Kim HA: Prologation of c-Jun N-terminal kinase is associated with cell death induced by tumor necrosis factor alpha in human chondrocytes. J Korean Med Sci 19: 567-573, 2004

26. Prasadam I, Mao X, Shi W, Crawford R and Xiao Y: Combination of MEK-ERK inhibitor and hyaluronic acid has a synergistic effect on anti-hypertrophic and pro-chondrogenic activities in osteoarthritis treatment. J Mol Med (Berl) 91: 369-380, 2013.

27. Bai X, Fan L, He T, Jia W, Yang L, Zhang J, Liu Y, Shi J, Su L and Hu D: SIRT1 protects rat lung tissue against severe burn-induced remote ALI by attenuating the apoptosis of PMVECs via $\mathrm{p} 38$ MAPK signaling. Sci Rep 5: 10277, 2015.

28. Becatti M, Taddei N, Cecchi C, Nassi N, Nassi PA and Fiorillo C: SIRT1 modulates MAPK pathways in ischemic-reperfused cardiomyocytes. Cell Mol Life Sci 69: 2245-2260, 2012. 